\title{
CRISPLD2 Gene Polymorphisms with Nonsyndromic Cleft Lip Palate in Indian Population
}

\author{
${ }^{1}$ Department of Orthodontics, Yenepoya University, Mangaluru, \\ Karnataka, India \\ ${ }^{2}$ Department of Craniofacial Surgery, GSR Institute of Craniofacial \\ Surgery, Hyderabad, Telangana, India \\ ${ }^{3}$ Department of Genetics, Vasavi Medical Research Centre, \\ Hyderabad, Telangana, India \\ Global Med Genet 2020;7:22-25.
}

Praveen Kumar Neela ${ }^{1,2}$ Srinivas Reddy Gosla ${ }^{2}$ Akhter Husain ${ }^{1}$ Vasavi Mohan ${ }^{3}$

Address for correspondence Praveen Kumar Neela, BDS, MDS, DIBO, M(Orth) RCPS (Glasg), Research Scholar, Department of Orthodontics, Yenepoya Dental College, Yenepoya University, Mangaluru, Karnataka 575018, India

(e-mail: praveenneela@yahoo.com).

\section{Introduction}

Cleft lip palate (CLP) is an important congenital disability affecting humans. An infant is born with a cleft lip and/or palate somewhere on the planet every 2 minutes according to a World Health Organization (WHO) study. ${ }^{1}$ Prevalence of cleft lip and palate varies significantly from one country to another. It is highest in North American Indians and East Asians (1:2,500) and lowest in Africans (1:500). The cleft incidence in India is around 1:800 to $1: 1,000$, and three infants are born with some type of cleft every hour. ${ }^{2}$ Cleft lip palate can be syndromic or nonsyndromic. A total of $70 \%$ of the cleft lip and palate cases are nonsyndromic, whereas 30\% are syndromic which are associated with some other anomalies. $^{3}$ Our understanding of the etiology and pathogenesis of nonsyndromic variants yet remains relatively poor. The etiology is multifactorial, ranging from genetic causes, malnutrition, endocrine disorders, infection, trauma, consanguinity, etc. Roughly $20 \%$ of the CLP showed consanguinity of their parents while the percentage of familial cases is $3.5 \%$ of all the cleft cases. ${ }^{4}$ Some form of cleft phenotype characterizes approximately 600 syndromes. ${ }^{5}$

\section{Genetic Causes}

Genetic research of clefts uses both association analysis and link analysis to determine the genetic determinants. The results of candidate gene-based association studies, performed on various ethnicities, populations have been mostly inconclusive or conflicting, with many candidate loci implicated in cleft phenotypes. Inconsistency is mostly due to genetic heterogeneity. Various researchers discovered multiple candidate genes linked to nonsyndromic CLP (NSCLP) such as IRF6, MSX1, ABC4, RARA, TGF $\alpha$, TGF $\beta$, p63, MYH9, BCL3, MTHFR, TGFB2, SATB2, P63, MSX2, FOXE1, BMP4, PAX7, PVRL1, TGFB3, RARA, RUNX2, BCL3, TGFB1, TBX1, and BCL3. ${ }^{6-14}$ Genetic variation in cysteine-rich secretory protein Limulus clotting factor $\mathbf{C}$, Cochlin (Coch-5b2) and Lgl1 (LCCL) domain containing 2 (CRISPLD2) gene reported as an etiological factor in CLP. ${ }^{15}$ Three SNPs identified in the study analyzed for its association in Northern Chinese published online July 8,2020
DOI https://doi.org/ 10.1055/s-0040-1713166. ISSN 2699-9404. (c) 2020 Georg Thieme Verlag KG Stuttgart · New York
License terms

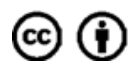


population revealed significant association. ${ }^{16}$ Studies conducted later also reported the involvement of this gene. ${ }^{17,18}$ Even studies on animal models also showed that it is an important gene associated with NSCLP. ${ }^{19}$

However, there is no reported data on the association of these SNPs in the Indian population. Therefore, the present study was aimed to evaluate the role of these high-risk SNPs in the etiology of CLP in Indian multiplex families of NSCLP.

\section{Materials and Methods}

\section{Ethical Approval of Research}

The Institutional Review Board of GSR Institute of Craniofacial Surgery, Hyderabad, India, approved this study. It is a highvolume cleft center in India. Multiplex families of Indian origin with NSCLP were selected. Patients with a monogenic syndrome or chromosomal aberrations, associated malformations, and mental retardation were excluded from the study. The control sample comprised of unaffected related individuals from these multiplex families. Based on the power calculation for family-based association studies, 20 multiplex families were selected. These include 1 family with five affected, 2 families with four affected, 5 families with three affected, and 12 families with two affected cleft patients. Four multigenerational families reported consanguinity. Informed consent obtained from all the participants. Parents' consent was taken when the affected participants were minors.

\section{DNA Isolation}

Venous blood of 4 to $5 \mathrm{~mL}$ was taken in the Ethylene Diamine Tetra Acetic acid (EDTA) tubes. Genomic DNA was extracted from the blood lymphocytes using the salting-out method. ${ }^{20}$ An ultraviolet spectrometer was used to calculate the average $260 / 280 \mathrm{~nm}$ ratio to assess the purity and concentration of DNA. The ratio of absorbance readings at the two wavelengths should be between 1.8 and 2.0 (i.e., A260/ A280 $=1.7-2.0$ ). Later, the DNA sent for SNP genotyping of the polymorphisms.

\section{Characteristics of the Single Nucleotide Polymorphism}

The characteristics of the selected polymorphisms rs1546124, rs4783099, and rs16974880 (-Table 1) are taken from web sites (www.ncbi.nlh.nih.gov/snp/ and http://asia.ensembl.org/ Homo_sapiens/Info/Index).

\section{Single Nucleotide Polymorphism Genotyping}

The SNP genotyping was done utilizing the Agena Bio MassARRAY (Agena Bioscience, Inc.; San Diego, California,
United States) platform using iPLEX Gold technology. This platform is a nonfluorescent, highly accurate detection platform utilizing Matrix-Assisted Laser Desorption/IonizationTime of Flight (MALDI-TOF) mass spectrometry. The assay was designed using proprietary Agena software (Assay Design Suite 2.0). The assay design was used to design primers. Follow the correct workflow according to the MassARRAY protocol, and finally run the sample through the analyzer. Agena's Spectro Typer 4.0 software (San Diego, California, United States) was used, which automatically generates reports that identify the SNP alleles (homozygous or heterozygous). The data obtained from the analyzer software is sent for statistical analysis.

\section{Data Analyses}

The SNP allele data of the affected and unaffected obtained from the MassARRAY system was subjected to statistical analysis. PLINK software (version 1.09) was used for this study. $^{21}$ It is an open-source whole-genome association toolset, which performs a varied range of analyses from basic-to-large scale. Genotype distribution was used to calculate the Hardy-Weinberg equilibrium (HWE). Comparisons between the affected and unaffected were performed using this PLINK software. Odds ratio (OR) and 95\% confidence intervals (CIs) were provided. Allelic association was analyzed using the Chi-square test. For nominal association, the statistical significance level is set to $\alpha=0.05$

\section{Results}

All the three SNPs present on CRISPLD2 were genotyped in 20 multiplex families. All the polymorphisms follow HardyWeinberg equilibrium. In the allele association analysis (-Table 2), we observed that none of the variants showed any association with NSCLP. None of them showed a $p$-value of $<0.05$. The OR also was less than 1.3.

\section{Discussion}

Development of craniofacial complex is one of the complex events during early phases of embryonic development, coordinated by a network of various transcription factors, and signaling molecules along with proteins conferring cell polarity and intercellular interactions. Any disturbance may result in failure to join the developmental process leading to multiple types of facial clefts. ${ }^{22}$ The importance of genomic research on the etiology of facial clefts is ever increasing. With advancements in the field of molecular biology, our envelope of research has grown. Identification of genetic

Table 1 Characteristics of nucleotide variants selected for the study

\begin{tabular}{|l|l|l|l|l|l|}
\hline Gene & Polymorphism & Type of alteration & Alleles & Ancestral allele & Global MAF \\
\hline CRISPLD2 & rs1546124 & 5 Prime UTR variant & G/A/C & C & 0.34 \\
\hline CRISPLD2 & rs4783099 & 3 Prime UTR variant & C/T & C & 0.39 \\
\hline CRISPLD2 & rs16974880 & 3 Prime UTR variant & T/A/G & T & 0.24 \\
\hline
\end{tabular}

Abbreviations: A, adenine; C, cytosine; G, guanine; MAF, minor allele frequency; T, thymine; UTR, untranslated region. 
Table 2 Association between CRISPLD2 polymorphisms and NSCLP

\begin{tabular}{|l|l|l|l|l|l|l|l|l|}
\hline SNP & BP & A1 & F_A & F_U & A2 & CHISQ & $p$-Value & OR (95\% Cl) \\
\hline rs1546124 & 16 & G & 0.17 & 0.1974 & C & 0.2174 & 0.641 & 0.84 \\
\hline rs4783099 & 17 & T & 0.27 & 0.2237 & C & 0.494 & 0.4821 & 1.29 \\
\hline rs16974880 & 24 & A & 0.12 & 0.1184 & T & 0.001024 & 0.9745 & 1.01 \\
\hline
\end{tabular}

Abbreviations: A, adenine; A1, major allele (wild allele); A2, minor allele (mutant); BP, base pairs; CHISQ, Chi-square; CHR, chromosome number; CI, confidence interval; F_U, minor allele frequency unaffected; F_A, minor allele frequency affected; G, guanine; NSCLP, nonsyndromic cleft lip plate; OR, odds ratio; SNP, single nucleotide polymorphism; $T$, thymine.

Note: the $p$-value $<0.05$ is significant.

polymorphisms in our population would be invaluable in understanding the developmental mechanisms involved in causing the disease. Data from animal models, in which clefts arise either spontaneously or as a result of mutagenesis experiment, combined with an analysis of how expression patterns correlate with gene function, and examining the effects of gene-environment interactions have proven themselves as powerful tools for identifying candidate genes for complex traits, like NSCLP. These animal model studies contribute to our knowledge of normal craniofacial development and the molecular pathogenesis of CLP, taking into account that facial development in mice mirrors human craniofacial development.

Research on various populations identified various candidate genes associated with CLP. Associations between SNPs in RUNX2, BMP4, TGFB3, PAX7, NTN1, IRF6, PTHFR, GHR, CRISPLD2, etc., and risk of clefts have been identified in different populations. Various genetic studies were conducted on diverse populations both on syndromic and nonsyndromic cases. Only IRF6 variants showed consistency in the etiology of CLP in different populations. Studies were conducted on case-parent trios, isolated clefts, and familial cases. In India, research on familial nonsyndromic cases is very less. Hence, the study was conducted on familial cases of NSCLP. Familial CLP corresponds to around 3.5\% of the total cleft cases. ${ }^{4}$ GSR Institute of Craniofacial surgery is a highvolume cleft center located in India. The sample population was taken from this center as patients from different parts of the country come for treatment. The nonsyndromic and familial cases were identified after a thorough medical history and examination of the patients.

CRISPLD2 is an essential gene involved in protein-coding and reported to be associated with cleft lip palate in Chinese, ${ }^{17,18}$ Irish population, ${ }^{23}$ and studies on animal models. ${ }^{19}$

Three polymorphisms, rs1546124, rs4783099, and rs16974880, were analyzed in the NSCLP as there is no reported literature on the role of these polymorphisms in the Indian population.

The results of the present study suggest that rs1546124, rs4783099, and rs16974880 are not significantly associated with NSCLP. In Northwestern Chinese population, SNP rs1546124 is significantly related to NSCLP whereas SNP rs4783099 was associated considerably to cleft palate only. ${ }^{17}$ In Uyghur population, rs1546124 was associated with NSCLP. ${ }^{18}$ In a meta-analysis, the authors concluded that rs4783099 induced a significantly increased risk for CLP. ${ }^{24}$
The variation or inconsistent association of these polymorphisms for the different populations/ethnicities in the etiology of CLP could be due to multifactorial, the difference in the ethnicity, epigenetic causes, and gene-to-gene interactions. Future research should focus on the additional markers on CRISPLD2, incorporation of isolated cases of clefts, and also on the functional role of these SNPs.

\section{Conclusion}

The results of this study indicate that among the multiplex families in India, the polymorphisms rs1546124, rs4783099, and rs16974880 of CRISPLD2 not associated with increased risk of NSCLP. Further studies are required to study the role of other SNPs on this gene and other candidate genes with bigger sample size.

\section{Funding}

None.

\section{Conflict of Interest}

None declared.

\section{Acknowledgment}

We would like to thank all the patients for their voluntary participation in this genetic study.

\section{References}

1 Mossey P. Global strategies to reduce the healthcare burden of craniofacial anomalies. Br Dent J 2003;195(10):613

2 Reddy SG, Reddy RR, Bronkhorst EM, et al. Incidence of cleft lip and palate in the state of Andhra Pradesh, South India. Indian J Plast Surg 2010;43(02):184-189

3 Dixon MJ, Marazita ML, Beaty TH, et al. Cleft lip and palate: understanding genetic and environmental influences. Nat Rev Genet 2011;12(03):167-178

4 Neela PK, Reddy SG, Husain A, et al. Association of cleft lip and/or palate in people born to consanguineous parents: A 13-year retrospective study from a very high-volume cleft center. J Cleft Lip Palate Craniofac Anomal 2019;6:33-37

5 Mossey P, Little J. Addressing the challenges of cleft lip and palate research in India. Indian J Plast Surg 2009;42:S9-S18

6 Sull JW, Liang KY, Hetmanski JB, et al. Differential parental transmission of markers in RUNX2 among cleft case-parent trios from four populations. Genet Epidemiol 2008;32(06):505-512

7 Beaty TH, Murray JC, Marazita ML, et al. A genome-wide association study of cleft lip with and without cleft palate identifies risk variants near MAFB and ABCA4. Nat Genet 2010;42(06):525-529 
8 Leslie EJ, Liu H, Carlson JC, et al. A genome-wide association study of nonsyndromic cleft palate identifies an etiologic missense variant in GRHL3. Am J Hum Genet 2016;98(04):744-754

9 Vieira AR. Unraveling human cleft lip and palate research. J Dent Res 2008;87(02):119-125

10 Letra A, Menezes R, Fonseca RF, et al. Novel cleft susceptibility genes in chromosome 6q. J Dent Res 2010;89(09):927-932

11 Mehrotra D. Genomic expression in non syndromic cleft lip and palate patients: A review. J Oral Biol Craniofac Res 2015;5(02): 86-91

12 Mohamad Shah NS, Salahshourifar I, Sulong S, et al. Discovery of candidate genes for nonsyndromic cleft lip palate through genome-wide linkage analysis of large extended families in the Malay population. BMC Genet 2016;17:39

13 Funato N, Nakamura M. Identification of shared and unique gene families associated with oral clefts. Int J Oral Sci 2017;9(02): 104-109

14 Bahrami R, Dastgheib SA, Niktabar SM, et al. Association of BMP4 rs17563 polymorphism with nonsyndromic cleft lip with or without cleft palate risk: literature review and comprehensive meta-analysis. Fetal Pediatr Pathol 2020;7:1-15

15 Chiquet BT, Lidral AC, Stal S, et al. CRISPLD2: a novel NSCLP candidate gene. Hum Mol Genet 2007;16(18):2241-2248

16 Shi J, Jiao X, Song T, et al. CRISPLD2 polymorphisms are associated with non-syndromic cleft lip with or without cleft palate in a northern Chinese population. Eur J Oral Sci 2010;118(04):430-433
17 Shen X, Liu RM, Yang L, et al. The CRISPLD2 gene is involved in cleft lip and/or cleft palate in a Chinese population. Birth Defects Res A Clin Mol Teratol 2011;91(10):918-924

18 Mijiti A, Ling W, Maimaiti A, et al. Preliminary evidence of an interaction between the CRISPLD2 gene and non-syndromic cleft lip with or without cleft palate (nsCL/P) in Xinjiang Uyghur population, China. Int J Pediatr Otorhinolaryngol 2015;79(02):94-100

19 Chiquet BT, Yuan Q, Swindell EC, et al. Knockdown of Crispld2 in zebrafish identifies a novel network for nonsyndromic cleft lip with or without cleft palate candidate genes. Eur J Hum Genet 2018;26(10):1441-1450

20 Miller SA, Dykes DD, Polesky HF. A simple salting out procedure for extracting DNA from human nucleated cells. Nucleic Acids Res $1988 ; 16(03): 1215$

21 Purcell S, Neale B, Todd-Brown K, et al. PLINK: a tool set for wholegenome association and population-based linkage analyses. Am J Hum Genet 2007;81(03):559-575

22 Stanier P, Moore GE. Genetics of cleft lip and palate: syndromic genes contribute to the incidence of non-syndromic clefts. Hum Mol Genet 2004;13(Spec No 1):R73-R81

23 Carter TC, Molloy AM, Pangilinan F, et al. Testing reported associations of genetic risk factors for oral clefts in a large Irish study population. Birth Defects Res A Clin Mol Teratol 2010;88(02):84-93

24 Ge X, Shi QM, Ding Z, et al. Association between CRISPLD2 polymorphisms and the risk of nonsyndromic clefts of the lip and/or palate: a meta-analysis. Cleft Palate Craniofac J 2018;55(03):328-334 